\title{
61.
}

\section{ON TCHEBYCHEFF'S THEORY OF THE TOTALITY OF THE PRIME NUMBERS COMPRISED WITHIN GIVEN LIMITS.}

\author{
[American Journal of Mathematics, IV. (1881), pp. 230-247.]
}

IF it be admitted that Legendre's approximate formula for the number of prime numbers inferior to a given number, which has been confirmed by direct enumeration of the number of prime numbers contained in the first few millions, can be extended to those remote regions of number which transcend the limits and even the possibilities of human experience, it will follow as a consequence that the average density of the distribution of prime numbers in the neighbourhood of a large quantity $x$ approximates to $\frac{1}{\log x}$, and consequently that the number of primes included between $x$ and $(1+\epsilon) x$, or if we like to say so, between $x+A$ and $(1+\epsilon) x+B$, will be approximately equal to $\frac{\epsilon x}{\log x}$, and therefore will become indefinitely great, however small $\epsilon$ may be taken. Although there can hardly be a doubt that such is the fact, no step had been taken previous to Tchebycheff's researches towards establishing this proposition demonstratively. Tchebycheff has succeeded in proving it, not, it is true, in an absolute sense, but for all values of $\epsilon$ exceeding the fraction $\frac{1}{5}$. He has done more, inasmuch as he has given formulae for actually ascertaining a number $x$ for all values superior to which there will be at least any specified number $K$ of primes included between $x+A$ and $(1+\epsilon) x+B$ when $\epsilon$ has any positive value superior to $\frac{1}{5}$, and $A$ and $B$ are any quantities positive or negative. He may not perhaps have actually stated this proposition in so many words, but it is an immediate inference from the limits (expressed in terms of $x, x^{\frac{1}{2}}$ and $\log x$ ) which he has obtained to the number of prime numbers not exceeding $x$. The object of what follows is to make a little further advance in the same 
direction, and to show upon Tchebycheff's own principles that the proposition remains true when $\epsilon$ is conditioned no longer to be inferior to the fraction $\frac{1}{5}$, but to the fraction $\frac{1}{6}+\frac{1}{4642 \frac{10}{11}}$, so that the excess above unity (the region so to say of darkness) is scarcely more than five-sixths of what it is for the first named fraction. This conclusion is arrived at by aid exclusively of Tchebycheff's own formulae.

Tchebycheff's method may be regarded as the first approximation to the inferior and superior limits of a quantity $\psi x$ subject to the conditions

$$
\begin{aligned}
& V x>A x+F \log x, \\
& V x<A x+F_{1} \log x,
\end{aligned}
$$

where

$$
V x=\psi x-\psi \frac{x}{6}+\psi_{7}^{x}-\psi \frac{x}{10} \text { etc., }
$$

(see Serret's Cours d'Algèbre supérieure, 4th Ed., Vol. II., pp. 230-233), and to the further conditions that $\psi x$ is not less than $\psi x^{\prime}$ if $x>x^{\prime}$, and that $\psi x=0$ when $x<1$.

The limits obtained for $\psi x$ depend exclusively on these definitions, and would be applicable to any function $\psi x$ whatever that satisfied them.

The advance made in this article consists in pursuing the approximation through an indefinite number of steps, so as to bring the superior and inferior limits to $\psi x$ continually nearer and nearer to each other as regards the principal term (a multiple of $x$ ) which enters into each of them: the remaining terms over and above this multiple of $x$ in the expressions for the limits always continue to be positive integer powers of $\log x$, and consequently the ratio of the limits becomes as nearly as we please identical with the ratio of the principal terms (that is of their coefficients) when $x$ is taken sufficiently great: this ratio as given in the first approximation is $\frac{6}{5}$, but as the approximation is continued continually converges to but never reaches the fraction

$$
\frac{7}{6}+\frac{1}{4642 \frac{10}{11}} .
$$

Such, and such only, is the small but not unimportant contribution here supplied to Tchebycheff's remarkable theory. As no allusion is made to the possibility of this contraction of the limits in a work published so recently as 1879 , by an author so competent as M. Serret, I presume that it has hitherto remained unnoticed; but of this I cannot speak with certainty, inasmuch as it was enough for M. Serret's purpose to obtain for the ratio of the principal terms a number less than 2 ; that being sufficient for the object he had in view, which was to prove M. Bertrand's celebrated postulate that at least one prime number must be included (for all values of $x$ greater than $\frac{7}{2}$ ) between $x$ and $2 x-2$. 
Although I might confine myself exclusively to the determination of the limits to $\psi x$ which flow from the conditions above given, it is, I think, desirable to supply a brief summary of M. Tchebycheff's method, so as to point out the connexion between the determination of these limits and the limits to "the totality of the prime numbers comprised within a given range." In so doing I shall adopt for the convenience of reference the notation which I find in M. Serret's able exposition of the subject (Alg. sup., Vol. II. pp. 225-239).

$\theta x$ stands for the sum of the logarithms of all the prime numbers not exceeding $x$.

$$
\begin{aligned}
& \psi x=\theta x+\theta x^{\frac{1}{2}}+\theta x^{\frac{1}{3}}+\theta x^{\frac{1}{4}}+\theta x^{\frac{1}{5}}+\ldots \\
& T x=\psi x+\psi \frac{x}{2}+\psi \frac{x}{3}+\psi \frac{x}{4}+\psi \frac{x}{5}+\ldots
\end{aligned}
$$

and, as a consequence founded on purely arithmetical considerations, $T x$ is the sum of the logarithms of all the numbers not exceeding $x$, and therefore, as an easy deduction from Stirling's theorem, it follows that for all values of $x$ superior to unity,

$$
\begin{aligned}
& T x<x \log x-x+\frac{1}{2} \log x+\left\{\log \sqrt{ }(2 \pi)+\frac{1}{12}\right\} \\
& T x>x \log x-x-\frac{1}{2} \log x+\log \sqrt{ }(2 \pi)
\end{aligned}
$$

If then $V x$ (a notation not in Serret) be used to denote

$$
T x-T \frac{x}{2}-T \frac{x}{3}-T \frac{x}{5}+T \frac{x}{30}
$$

(where it should be noticed that $1-\frac{1}{2}-\frac{1}{3}-\frac{1}{5}+\frac{1}{30}=0$ ), limits for $V x$ can be found in which $x \log x$ will not appear, and expressed solely in terms of $x$ and $\log x$ : it may in fact be shown that for all values of $x$ superior to unity,

$$
\begin{aligned}
& V x>A(x-1)-\frac{5}{2} \log x \\
& V x<A(x-1)+\frac{5}{2} \log x,
\end{aligned}
$$

where $\quad A=\frac{1}{2} \log 2+\frac{1}{3} \log 3+\frac{1}{5} \log 5-\frac{1}{30} \log 30=\cdot 92129202 \ldots$

The limits actually employed, however, are the slightly wider ones,

$$
\begin{aligned}
& V x>A x-\frac{5}{2} \log x-1 \\
& V x<A x+\frac{5}{2} \log x .
\end{aligned}
$$


If now we take an infinite succession of numbers separable into batches of sixteen, such that every $(i+1)$ th batch may be got by adding $30 i$ to each of the numbers in the first batch, those numbers being

$$
1,6,7,10,11,12,13,15,17,18,19,20,23,24,29,30
$$

(where it is perhaps worth noticing that leaving out the last number 30 , the remaining 15 consist of a middle term 15 and pairs of numbers whose sum is always 30 , disposed symmetrically about that middle term), it will readily be seen to follow from the expression for $V$ in terms of the $T$ 's and of $T$ in terms of the $\psi$ 's, that

$$
\left.\begin{array}{rl}
V x & =\psi x-\psi \frac{x}{6}+\psi \frac{x}{7}-\psi \frac{x}{10}+\psi \frac{x}{11}-\psi \frac{x}{12}+\psi \frac{x}{13}-\psi \frac{x}{15} \\
& +\psi \frac{x}{17}-\psi \frac{x}{18}+\psi \frac{x}{19}-\psi \frac{x}{20}+\psi \frac{x}{23}-\psi \frac{x}{24}+\psi \frac{x}{29}-\psi \frac{x}{30}
\end{array}\right\}
$$

just in the same way as if supposing $\omega x=\psi x-2 \psi \frac{x}{2}$ we should find

$$
\omega x=\psi x-\psi \frac{x}{2}+\psi \frac{x}{3}-\psi \frac{x}{4}+\psi \frac{x}{5} \ldots ;
$$

or as if supposing $\Omega x=\psi x-\psi \frac{x}{2}-\psi \frac{x}{3}-\psi \frac{x}{6}$ we should find

$$
\Omega x=\psi x+\psi \frac{x}{5}-2 \psi \frac{x}{6}+\psi \frac{x}{7}+\psi \frac{x}{11}-2 \psi \frac{x}{12}+\ldots .
$$

From the limits to which $V x$ is subject ( $V x$ being now regarded as representing the series of $\psi$ 's above written) limits can be found to $\psi x$ of the form $m x+R_{1}(\log x), n x+R_{2}(\log x)$, where the $R$ 's signify rational integer forms of function. In the first approximation, for the inferior and superior limits respectively, $m=A, n=6 \frac{A}{5} ; R_{1}$ is a linear and $R_{2}$ a quadratic form of function. In the approximation of the $i$ th order $m$ and $n$ will become functions of $i$, and $R_{1}, R_{2}$ will be of the $i$ th and $(i+1)$ th orders respectively in $\log x$.

The limits of $\psi x$ being supposed to be given (say $\psi^{\prime} x$ the superior and $\psi_{1} x$ the inferior limit), $\psi^{\prime} x$ will serve as a superior and $\psi_{1} x-2 \psi^{\prime} x^{\frac{1}{2}}$ as an inferior limit to $\theta x$. But instead of $\psi^{\prime} x$ we may use (although not at all 
necessary for the object in view) the slightly closer limit $\psi^{\prime} x-\psi_{1} x^{\frac{1}{2}}$, which is what M. Serret employs, and equally instead of $\psi_{1} x-2 \psi^{\prime} x^{\frac{1}{2}}$ we might use the slightly closer limit

$$
\psi_{1} x-\psi^{\prime} x^{\frac{1}{2}}-\psi^{\prime} x^{\frac{1}{3}}-\psi^{\prime} x^{\frac{1}{5}}+\psi_{1} x^{\frac{1}{30}}
$$

which, probably as leading to calculations needlessly complicated (as regards the object in view), M. Serret does not employ. In any case, following the same notation as before to distinguish the two limits, we shall obtain

$$
\begin{aligned}
& \theta^{\prime} x=n x+F^{\prime}\left(x^{\frac{1}{2}}, \log x\right), \\
& \theta_{1} x=m x+F^{\prime}(\ldots, \log x),
\end{aligned}
$$

where $F, F^{\prime}$ are rational integer forms of function, and the dots in the $F^{\prime \prime}$ may be filled in either with $x^{\frac{1}{2}}$ or with $x^{\frac{1}{2}}, x^{\frac{1}{3}}, x^{\frac{1}{5}}, x^{\frac{1}{30}}$; and we shall have

$$
\theta^{\prime} x=n x\left(1+\epsilon_{x}\right), \quad \theta_{1} x=m x\left(1+\eta_{x}\right),
$$

where $\epsilon_{x}$ and $\eta_{x}$ vanish when $x=\infty$.

To come to our ultimate object, it is obvious that the number of primes between $x$ and $(1+\rho) x$ will be greater than $\left[\theta_{1}(1+\rho) x-\theta^{\prime} x\right] \div \log x$. It will therefore be greater than $\frac{[m(1+\rho)-n] x+\delta_{x}}{\log x}$, where $\delta_{x}=0$ when $x=\infty$. Hence we may find a value of $x$ so great that the number of primes shall be at least $K$ by finding a number $x$ sufficiently large to make

$$
\theta_{1}(1+\rho) x-\theta^{\prime} x-(K-1) \log x>0,
$$

which it must always be possible to do provided that $m(1+\rho)>n$, that is, that $\rho>\left(\frac{n}{m}-1\right)$. Hence the importance of diminishing what I call the asymptotic ratio $\frac{n}{m}$, that is, the ratio of the coefficients in the principal terms of the superior and inferior limits to $\psi x$. That is what I shall now proceed to accomplish, but first it is necessary to establish a certain easy lemma.

Suppose the equation $f x-f \frac{x}{c}=A x^{m}$ is to be satisfied; this can be done by writing $f x=A \frac{c^{m}}{c^{m}-1} x$, and in particular if $m=1$, the only case that the present theory demands, $f x=\frac{c}{c-1} A x$. Again if the equation

$$
f x-f \frac{x}{c}=P(\log x)^{\mu}
$$

is to be satisfied, this may be done by making

$$
f x=P_{0}(\log x)^{\mu+1}+P_{1}(\log x)^{\mu}+P_{2}(\log x)^{\mu-1}+\ldots+P_{\mu} \log x,
$$

for since $\log \frac{x}{c}=(\log x-\log c), f x-f \frac{x}{c}$ will then obviously become a function 
of $\log x$ of the $\mu$ th order, which may be identified with $P(\log x)^{\mu}$ by properly assigning the values of the $(\mu+1)$ disposable constants $P_{0}, P_{1}, P_{2}, \ldots P_{\mu}$. In fact the equation might easily (if it were worth while to do so) be turned into an equation of differences, and the general values of the $P$ 's be expressed once for all in terms of Bernoulli's numbers for any value of $\mu$. Hence it follows that the equation

$$
f x-f \frac{x}{c}=N x+R_{\mu}^{*} \log x,
$$

where $R_{\mu}$ is a rational integer form of function of the $\mu$ th order, may be satisfied by making

$$
f x=\frac{c}{c-1} N x+R_{\mu+1} \log x,
$$

where the second term on the right hand side of the equation is a known function of $\log x$ of the $(\mu+1)$ th order.

Suppose now that the inequality $\psi x-\psi \frac{x}{c}<N x+R_{\mu} \log x$, where $c>1$, is given, and it is desired to extract from this inequality an inferior limit to $\psi x$. It is only necessary to get a solution of the equation

$$
f x-f \frac{x}{c}=N x+R_{\mu} \log x .
$$

We shall then have

$$
\begin{aligned}
& \psi x-\psi \frac{x}{c}<f x-f \frac{x}{c} \\
& \psi \frac{x}{c}-\psi \frac{x}{c^{2}}<f \frac{x}{c}-f \frac{x}{c^{2}} \\
& \psi \frac{x}{c^{2}}-\psi \frac{x}{c^{3}}<f \frac{x}{c^{2}}-f \frac{x}{c^{3}}
\end{aligned}
$$

and consequently

$$
f x-f \frac{x}{c^{q}}>\psi x-\psi \frac{x}{c^{q}} .
$$

If then $q$ be supposed to be taken such that $\frac{x}{c^{q}}$, say $z$, lies between 0 and 1 , we shall have

$$
f x-\psi x>f z
$$

and $\dot{a}$ fortiori $>R_{\mu+1} \log z$ (if $N$ be positive, as is the case throughout the present investigation), where the right hand side of the inequality is a known rational integer function of $\log z$. If then $M$ be a number less than the least value that $R_{\mu+1} \xi$ can assume between the limits $\xi=0, \xi=-\log c$, we shall have $\psi x<f x-M$, and an inferior limit will have been obtained to $\psi x$.

* The reader's attention is called to the fact that $R_{\mu}$ is used throughout to denote a form of function, and not, like $P_{\mu}$, a coefficient. 
In the first approximation (Serret, p. 234), where $\mu=1$ and $c=6$,

$$
R_{2} \xi=\frac{5}{4 \log 6} \xi^{2}+\frac{5}{4} \xi
$$

the minimum value of which is got by taking $2 \xi=-\log 6$ or $\xi=-\log \sqrt{ } 6$ (which happens to lie between the limits of $\log 1$ and $-\log 6$ ) and gives $M=\frac{-5 \log 6}{16}$, so that $\psi x<f x+\frac{5 \log 6}{16}$. The actual value employed for the superior limit, as sufficiently near and more convenient for use, is $f x+1$.

So in the general case we shall have $f x-\psi x>M$ where $M$ is any number less than the least value of $R_{i+1} \xi$ for values of $\xi$ lying between 0 and $-\log c$. It may or may not be the absolute minimum of $R_{i+1} \xi$ that has to be taken according as the value of $\xi$ which gives this absolute minimum does or does not lie between 0 and $-\log c$. In the latter case it may be either some other minimum, or one of the values of $R_{i+1} \xi$ corresponding to the extreme values $\xi=0$ and $\xi=-\log c$, which might be found by trial. But a method practically better and sufficient for the demands made by the present investigation, would be to substitute zero in place of any term in the function of $\xi$ of the form $+K \xi^{2 m}$ or $-K \xi^{2 m+1}$, and for any term of the form $-K \xi^{2 m}$ or $+K \xi^{2 m+1}$ to substitute $-K(\log c)^{2 m}$ and $-K(\log c)^{2 m+1}$ respectively.

For instance, in the case just considered we might have written

$$
M=-\frac{5}{4} \log 6,
$$

and the superior limit instead of being $f x+1$ would have been $f x+\frac{5}{4} \log 6$, which would practically have been just as good. With a view to a remark which will subsequently be made it is well to notice that the inequality

$$
\psi x-\psi \frac{x}{c}>N x+R_{\mu} \log x
$$

may also be solved precisely in the same manner, and will give for an inferior limit to $\psi x$ (using $f x$ to signify the very same function as before) $f x-M_{1}$, where ( $N$ being supposed positive) $M_{1}=-N \log c+$ any quantity not less than the greatest value of a known rational integer function of a variable conditioned to lie between 0 and $-\log c$, which may either be found by an exact algebraical process or by substituting 0 in those two cases where previously $-\log c$, and $-\log c$ in those other two cases where previously 0 was to be substituted for the variable.

The lemma needful for our purposes may now accordingly be stated in the following terms: If $\psi x-\psi \frac{x}{c}$ is less or greater than $N x+$ a given rational integer function of $\log x$ of any given order, $\psi x$ is less or greater than $\frac{c}{c-1} N x+$ a known (and easily determinable) rational integer function of $\log x$ of the order next superior. 
If the coefficients of $x$ in the superior and inferior limits to $\psi x$ at any stage of the investigation be called $u$ and $v$, I shall show that these values will serve to give (step by step) other superior and inferior limits where $u$ and $v$ are replaced by quantities $u^{\prime}, v^{\prime}$, such that $u^{\prime}<u, v^{\prime}>v ; u^{\prime}, v^{\prime}$ being known linear functions of $u, v$. We shall thus be led to a system of two simultaneous linear equations of differences in order to obtain the effect of those changes repeated any number, finite or infinite, of times: but for greater clearness I shall begin with supposing that one of the two expressions $u, v$, namely, $v$ (which undergoes far less modification than the other) is kept constant. There will then result a single scheme of successive substitutions leading to the construction of a single linear equation in differences.

The first step will then be as follows:

$$
\begin{aligned}
& \psi x-\psi \frac{x}{6}<A x+\frac{5}{2} \log x-\psi \frac{x}{7}+\psi \frac{x}{10} \\
& <A x+\frac{5}{2} \log x-\left(A \frac{x}{7}-\frac{5}{2} \log \frac{x}{7}-1\right)+\frac{6}{50} A x+\frac{5}{4 \log 6}\left(\log \frac{x}{10}\right)^{2}+\frac{5}{4} \log \frac{x}{10} \\
& \text { or writing } \quad \lambda=\log 6, \quad \mu=\log 7, \quad \nu=\log 10 \text {, } \\
& \psi x-\psi \frac{x}{6}<\frac{171}{175} A x+\frac{5}{4 \lambda}(\log x)^{2}+\left\{\left(\frac{25}{4}-\frac{5 \nu}{2 \lambda}\right) \log x+\frac{5 \nu^{2}}{4 \lambda}-\frac{5}{2} \mu-\frac{5}{4} \nu+1\right\} . \\
& \psi x<\frac{1026}{875} A x+P(\log x)^{3}+Q(\log x)^{2}+R x-M,
\end{aligned}
$$

where first to find $P, Q, R$, we have the three equations

$$
\begin{aligned}
3 P \lambda & =\frac{5}{4 \lambda} \\
-3 P \lambda^{2}+2 Q \lambda & =\frac{25}{4}-\frac{5 \nu}{2 \lambda} \\
P \lambda^{3}-Q \lambda^{2}+R \lambda & =\frac{5 \nu^{2}}{4 \lambda}-\frac{5}{2} \mu-\frac{5}{4} \nu+1
\end{aligned}
$$

that is, $P=\frac{5}{12 \lambda^{2}} ; \quad Q=\frac{15}{4 \lambda}-\frac{5 \nu}{4 \lambda^{2}} ; \quad R=-\frac{5}{12}+\frac{15}{4}-\frac{5 \nu}{2 \lambda}+\frac{5 \nu^{2}}{4 \lambda^{2}}-\frac{5 \mu}{2 \lambda}+\frac{1}{\lambda}$.

Here $P$ is positive; $Q$, whose sign depends on that of $3-\frac{\log 10}{\log 6}$, is also positive; and

$$
\begin{aligned}
R & =\frac{10}{3}+5\left(\frac{\nu}{2 \lambda}-\frac{1}{2}\right)^{2}-\frac{5 \mu-2}{2 \lambda}-\frac{5}{4} \\
& =3 \cdot 33333 \ldots+\cdot 10160 \ldots-2 \cdot 1570 \ldots-1 \cdot 25 \\
& =3 \cdot 43493 \ldots-3 \cdot 4070 \ldots, \text { which is also positive. }
\end{aligned}
$$

Hence we may make

$$
M=-P \lambda^{3}-R \lambda,
$$

or

$$
-M=1+\frac{15}{4} \lambda-\frac{5(\mu+\nu)}{2}+\frac{5 \nu^{2}}{4 \lambda}=1 \cdot 2947 \text {. }
$$


It is quite possible, and even most likely, that the minimum of

$$
P \lambda^{3}-Q \lambda^{2}+R \lambda
$$

(within the prescribed limits) would be found to exceed -1 were it worth while to go through the arithmetical calculations necessary to obtain it, but it is quite sufficiently near for all practical purposes to use the value above determined, or even to take $-M$ as great as 2 and to adopt for our new superior limit

$$
\frac{171}{175} A x+P(\log x)^{3}+Q(\log x)^{2}+R \log x+2 .
$$

In like manner this new limit will enable us to find another, and it is obvious that the general form of the limit obtained after $i$ of these steps have been gone through will be $u_{i} A x+R_{i+2} \log x$, where

$$
u_{i}=\frac{6}{5}\left(1-\frac{1}{7}+\frac{u_{i-1}}{10}\right), \text { that is, } u_{i}-\frac{3 u_{i-1}}{25}=\frac{36}{35} \text {. }
$$

Putting

and making

we have

Hence

$$
u_{i}=\omega_{i}+h
$$$$
\frac{22}{25} h=\frac{36}{35}, \text { that is, } h=\frac{90}{77} \text {, }
$$

$$
\begin{aligned}
& \omega_{i}-\frac{3}{25} \omega_{i-1}=0 . \\
& u_{i}=C\left(\frac{3}{25}\right)^{i}+\frac{90}{77}
\end{aligned}
$$

The ultimate value of $u_{i}$ is therefore $\frac{90}{77}$, and accordingly, by repeating the process indicated a sufficient number of times, we shall have for a superior limit $\left(\frac{90}{77}-\epsilon_{i}\right) A x+R_{i+2} \log x$, where $\epsilon_{i}$ may be made as small as we please by taking $i$ sufficiently great, and thus the ultimate asymptotic ratio of the two limits is $\frac{90}{77}$ instead of $\frac{6}{5}$.

Another mode of approximation may be used, as shown in what follows.

Since

$$
\psi x-\psi \frac{x}{10}<A x-\psi \frac{x}{6}+\psi \frac{x}{7}
$$

if we have found

$$
\psi x<u_{i}^{\prime} A x+R_{i+2} \log x
$$

we shall have $\psi x-\psi \frac{x}{10}<A x+u_{i}^{\prime} A \frac{x}{6}-A \frac{x}{7}+R_{i+2}^{\prime} \log x$,

and therefore

$$
\psi x<u_{i+1}^{\prime} A x+R_{i+3} \log x,
$$

where

$$
u_{i+1}^{\prime}=\frac{10}{9}\left\{1-\frac{1}{7}+\frac{1}{6} u_{i}^{\prime}\right\}
$$

that is,

$$
u_{i+1}^{\prime}-\frac{5}{27} u_{i}^{\prime}=\frac{20}{21} \text {; }
$$


or

$$
u_{i}^{\prime}=K\left(\frac{5}{27}\right)^{i}+h^{\prime}
$$

where

$$
h^{\prime}=\frac{27}{22} \cdot \frac{20}{21}=\frac{90}{77} \text {. }
$$

Thus $h^{\prime}=h$ and consequently also, if we suppose each of the two sorts of approximation to start from the same point, $K=C$.

Hence the ultimate value of $u_{i}$ and $u_{i}^{\prime}$ is the same, but the former method of approximation is to be preferred, as the same number of steps, that is, the same value of $i$, makes $C\left(\frac{5}{27}\right)^{i}+h$ always $>C\left(\frac{3}{25}\right)^{i}+h$. The corresponding values of $u_{i}, u_{i}^{\prime}$ have the same initial and final values, but for every intermediate value of $i, u_{i}<u_{i}{ }^{\prime}$. In fact $u_{i}, u_{i}^{\prime}$ are ordinates to the same abscissa of two non-intersecting curves, having a common starting point and a common asymptote.

The maximum value of $u_{i}^{\prime}-u_{i}$ is found by making $\left(\frac{5}{27}\right)^{i}-\left(\frac{3}{25}\right)^{i}$ a maximum, which takes place when $i$ is the integer next above or next below the value

$$
\frac{\log \log \frac{25}{3}-\log \log \frac{27}{5}}{\log \frac{25}{3}-\log \frac{27}{5}}, \text { which is obviously less than unity. }
$$

Hence after the first approximation $u_{i}$ and $u_{i}^{\prime}$ are always drawing closer together.

We may now proceed to the more (but only very slightly more) advantageous method of approximation, namely, that in which the principal terms in both limits are simultaneously varied, decreasing as before in the superior, and now at the same time increasing in the inferior limit.

Suppose then that we have found

$$
\begin{aligned}
& \psi x<u_{i} A x+R_{i+2} \log x \\
& \psi x>v_{i} A x+R_{i+1} \log x
\end{aligned}
$$

observing that $\frac{v_{i}}{24}-\frac{u_{i}}{29}$ is always positive, we shall succeed in increasing the principal term of the inferior limit by writing

$$
\psi x>A x+v_{i} A \frac{x}{24}-u_{i} A \frac{x}{29}+R_{i+2} \log x,
$$

and slightly more than previously diminishing the principal term in the superior limit by writing

$$
\psi x-\psi \frac{x}{6}<A x-v_{i} A \frac{x}{7}+u_{i} A \frac{x}{10}+R_{i+2}^{\prime} \log x .
$$


We shall thus easily derive

$$
\begin{aligned}
& \psi x>v_{i+1} A x+R_{i+2} \log x \\
& \psi x<u_{i+1} A x+R_{i+3} \log x
\end{aligned}
$$

where

$$
\begin{gathered}
v_{i+1}=1+\frac{v_{i}}{24}-\frac{u_{i}}{29} \\
u_{i+1}=\frac{6}{5}\left(1-\frac{v_{i}}{7}+\frac{u_{i}}{10}\right)=\frac{6}{5}-\frac{6}{35} v_{i}+\frac{3}{25} u_{i}
\end{gathered}
$$

or, making

$$
\begin{aligned}
& v_{i}=v_{i}^{\prime}+f, \quad u_{i}=u_{i}^{\prime}+e, \\
& v_{i+1}^{\prime}-\frac{1}{24} v_{i}^{\prime}+\frac{1}{29} u_{i}^{\prime}=0 \\
& u_{i+1}^{\prime}-\frac{3}{25} u_{i}^{\prime}+\frac{6}{35} v_{i}^{\prime}=0,
\end{aligned}
$$

if

$$
\frac{23}{24} f+\frac{1}{29} e=1, \quad \frac{6}{35} f+\frac{22}{25} e=\frac{6}{5} .
$$

So that, calling $\rho_{1}, \rho_{2}$ the roots of $\left|\begin{array}{rl}\rho-\frac{1}{24} ; & \frac{1}{29} \\ \frac{6}{35} ; & \rho-\frac{3}{25}\end{array}\right|=0$,

$$
\begin{aligned}
& u_{i}=C_{1} \rho_{1}^{i}+C_{2} \rho_{2}^{i}+e \\
& v_{i}=C_{1}^{\prime} \rho_{1}^{i}+C_{2}^{\prime} \rho_{2}^{i}+f .
\end{aligned}
$$

The equation for finding $\rho_{1}, \rho_{2}$ is

$$
\rho^{2}-\frac{97}{600} \rho-\frac{37}{40600}=0
$$

whence

$$
\rho_{1}=\cdot 167253 \ldots, \quad \rho_{2}=\cdot 005637 \ldots
$$

Also the equations in $e, f$ give $e, f$ (the values of $u_{\infty}, v_{\infty}$ ) as follows:

$$
e=\frac{59595}{50999}, \quad f=\frac{51072}{50999} .
$$

If there were any use in obtaining the values of the disposable constants they could of course be obtained from the equations

$$
\begin{array}{ll}
C_{1}+C_{2}+e=u_{0}=\frac{6}{5}, & C_{1} \rho_{1}+C_{2} \rho_{2}+e=u_{1}=\frac{1026}{875} \\
C_{1}^{\prime}+C_{2}^{\prime}+f=v_{0}=1, & C_{1}^{\prime} \rho_{1}+C_{2}^{\prime} \rho_{2}+f=v_{1}=\frac{3481}{3480} .
\end{array}
$$

The asymptotic ratio of the two limits is

$$
\frac{e}{f}=\frac{59595}{51072}=\frac{6}{7}+\frac{11}{51072} .
$$


Various other modes of approximation may be adopted, but it will be found that no smaller value can be obtained for the asymptotic ratio than that above given: the value of $u_{\infty}$ cannot be made less than $\frac{59595}{50999}$, nor the value of $v_{\infty}$ greater than $\frac{51072}{50999}$.

Thus for example, making use of the inequality

$$
\psi x-\psi \frac{x}{6}>A x-\psi \frac{x}{7}+\psi \frac{x}{24}-\psi \frac{x}{29}+R(\log x)
$$

we might by the lemma obtain

$$
\psi x>\frac{6}{5} A\left(1-\frac{u_{i}}{7}-\frac{u_{i}}{29}+\frac{v_{i}}{24}\right)+R_{i+3} \log x
$$

and consequently $\quad v_{i+1}=\frac{6}{5}\left(1-\frac{u_{i}}{7}-\frac{u_{i}}{29}+\frac{v_{i}}{24}\right)$;

combining which with the previous equation for $u_{i+1}$ we should have for finding $u_{\infty}, v_{\infty}$, say $e^{\prime}, f^{\prime}$, the two equations,

$$
\begin{aligned}
& \frac{19}{24} f^{\prime}+\frac{36}{203} e^{\prime}=1, \\
& \frac{6}{35} f^{\prime}+\frac{22}{25} e^{\prime}=\frac{6}{5},
\end{aligned}
$$

and consequently $\quad e^{\prime}=\frac{331905}{284029}, \quad f^{\prime}=\frac{284424}{284029}$.

Reduced to decimals

$$
\begin{array}{cl}
e=1 \cdot 16855 \ldots, & f=1 \cdot 00143 \ldots, \\
e^{\prime}=1 \cdot 16856 \ldots, & f^{\prime}=1 \cdot 00125 \ldots
\end{array}
$$

It may be noticed that $e A=1 \cdot 006774 \ldots, f A=\cdot 992619 \ldots$ of which the sum is nearly 1.999394 , and their mean nearly 999697 , whereas the mean of $A$ and $\frac{6 A}{5}$ (the original coefficients of $x$ in the limits) is nearly 1.01342 . Thus the new mean is more than 44 times nearer than the latter to the true asymptotic value deducible from the empirical formula.

Were it desired merely to find superior and inferior limits to $\psi x$ in the form obtained in Tchebycheff's method, it would (as already indicated) have been sufficient to have taken for $V x, T x-2 T \frac{x}{2}$, which would have led to the inequalities

$$
\begin{aligned}
& \psi x>(\log 2) x+R_{1} \log x, \\
& \psi x<2(\log 2) x+R_{2} \log x,
\end{aligned}
$$

but the asymptotic ratio being here 2 , these limits could not have conducted 
to a proof of M. Bertrand's postulate. If, however, we were to take $V x=T x-T \frac{x}{2}-T \frac{x}{3}-T \frac{x}{6}$ we should obtain

$$
\begin{aligned}
& V x>B x+R_{1} \log x, \\
& V x<B x+R_{1}^{\prime} \log x,
\end{aligned}
$$

where

$$
B=\frac{1}{2} \log 2+\frac{1}{3} \log 3+\frac{1}{6} \log 6=1 \cdot 0114043,
$$

and

$$
V x=\psi x+\psi \frac{x}{5}-2 \psi \frac{x}{6}+\psi \frac{x}{7}+\psi \frac{x}{11}-2 \psi \frac{x}{12}+\ldots,
$$

when we should obtain

$$
\begin{aligned}
& \psi x-\psi \frac{x}{6}<B x+R_{1}^{\prime} \log x \\
& \psi x<\frac{6}{5} B x+R_{2} \log x
\end{aligned}
$$

and again

$$
\begin{aligned}
& \psi x+\psi \frac{x}{5}>B x+R_{1} \log x \\
& \psi x>B\left(1-\frac{6}{25}\right) x+R_{2}^{\prime} \log x
\end{aligned}
$$

Here the asymptotic ratio of the two limits is $\frac{30}{19}$, which being less than 2 , the formulae above indicated would suffice to prove M. Bertrand's postulate, and would lead to an equation somewhat simpler in form than that led to by M. Tchebycheff's process, but whose greatest root would be considerably larger than that found by the established method; so that there would be a larger number of verifications of the postulate to be made for the lower numbers : this, however, is really a matter of very trifling importance, as the needful verifications could be made even up to 100,000 if necessary, by throwing a rapid glance over a few leaves of Burckhardt's tables.

It is noticeable that the limits above found by giving $V x$ the form $T x-2 T \frac{x}{2}$ are the only limits that can be got in such case; no process of successive approximation being here possible, on account of the too close contiguity of the successive denominators in $\psi x-\psi \frac{x}{2}+\psi \frac{x}{3} \ldots$

Such, however, would not be the case were we to use $V x$ to signify $T x-T \frac{x}{2}-T \frac{x}{3}-T \frac{x}{6}$, and consequently

$$
V x=\psi x+\psi \frac{x}{5}-2 \psi \frac{x}{6}+\psi \frac{x}{7}+\psi \frac{x}{11}-2 \psi \frac{x}{12}+\psi \frac{x}{13} \ldots
$$


The limits expressed by the inequalities

$$
\begin{aligned}
& \psi x<u_{i} B x+\ldots, \\
& \psi x>v_{i} B x+\ldots,
\end{aligned}
$$

would lead to the narrower limits

$$
\begin{aligned}
& \psi x<u_{i+1} B x+\ldots, \\
& \psi x>v_{i+1} B x+\ldots,
\end{aligned}
$$

where

$$
\begin{array}{r}
u_{i+1}=\frac{6}{5}\left(1-\frac{v_{i}}{7}+\frac{u_{i}}{12}\right), \\
v_{i+1}=1-\frac{u_{i}}{5}+\frac{v_{i}}{6}-\frac{u_{i}}{11}, \\
u_{i+1}-\frac{u_{i}}{10}+\frac{6 v_{i}}{35}-\frac{6}{5}=0, \\
\frac{u_{i}}{5}+\frac{u_{i}}{11}+v_{i+1}-\frac{v_{i}}{6}-1=0 .
\end{array}
$$

that is to say

Hence, using as before $e, f$ to indicate the ultimate values of $u_{i}, v_{i}$, we should have

$$
\begin{array}{r}
21 e+4 f-28=0, \\
96 e+275 f-330=0,
\end{array}
$$

and consequently

$$
e=\frac{6380}{5391}, \quad f=\frac{4242}{5391},
$$

and

$$
\frac{e}{f}=\frac{6380}{4242}=\frac{3}{2}+\frac{1}{249 \frac{9}{17}},
$$

which is the ultimate value of the asymptotic ratio, of which the initial value was $\frac{30}{19}$, that could be found by this method.

In every such kind of series as I have denoted by $V x$, it is obvious that the sum of the multiples of $x$ under the sign of $\psi$ in $V x$ is equal to the coefficient of $x$ in either limit to $V x$. Thus, for example, in Tchebycheff's series, if we take $n$ a multiple of 30 , and make $S_{n}=1+\frac{1}{2}+\frac{1}{3}+\ldots+\frac{1}{n}$, the sum of $n$ terms of $1-\frac{1}{6}+\frac{1}{7}-\frac{1}{10}+\frac{1}{11} \ldots$

$$
\begin{aligned}
& =\left(1-\frac{1}{2}-\frac{1}{3}-\frac{1}{5}+\frac{1}{30}\right) S_{n}+\frac{1}{2}\left(\frac{1}{\frac{1}{2} n+1}+\frac{1}{\frac{1}{2} n+2}+\ldots+\frac{1}{n}\right) \\
& +\frac{1}{3}\left(\frac{1}{\frac{1}{3} n+1}+\frac{1}{\frac{1}{3} n+2}+\ldots+\frac{1}{n}\right)+\frac{1}{5}\left(\frac{1}{\frac{1}{5} n+1}+\frac{1}{\frac{1}{5} n+2}+\ldots+\frac{1}{n}\right) \\
& -\frac{1}{30}\left(\frac{1}{\frac{1}{30} n+1}+\frac{1}{\frac{1}{30} n+2}+\ldots+\frac{1}{n}\right)
\end{aligned}
$$


and the multiplier of $S_{n}$ being always 0 , it follows that the sum of an infinite number of the consecutive terms

$$
=\frac{1}{2} \log 2+\frac{1}{3} \log 3+\frac{1}{5} \log 5-\frac{1}{30} \log 30=A .
$$

It may not unreasonably be conjectured that whilst nothing more can be done with the Tchebycheffian $V x$, it may be possible to find such other form of function in lieu of it, or such infinite succession of different forms of function, as may either directly or by successive approximation bring the coefficients of $x$ in the two limits as near as we please to one another, at the expense, of course, of proportionally lengthening out the residues, or tails as they might be termed, of the two limits. Could this be done, it is easy to demonstrate that the limit thus continually approached from opposite sides must be unity, as indicated in advance by Legendre's empirical formula. For this purpose it will be sufficient to use the simplest form of $V x$, namely, $T x-2 T \frac{x}{2}$, whence we obtain

$$
\begin{aligned}
& \psi x-\psi \frac{x}{2}+\psi \frac{x}{3} \ldots>\log 2 . x\left(1+\epsilon_{x}\right), \\
& \psi x-\psi \frac{x}{2}+\psi \frac{x}{3} \ldots<\log 2 . x\left(1+\eta_{x}\right),
\end{aligned}
$$

$\epsilon_{x}, \eta_{x}$ being known logarithmic quantities which vanish when $x=\infty$.

For suppose it possible to prove that, with a value of $h$ capable of being made less than any assignable quantity,

$$
\begin{aligned}
& \psi x>Q(1-h) x+G x, \\
& \psi x<Q(1+h) x+F x,
\end{aligned}
$$

where $\frac{F x}{x}, \frac{G x}{x}$ may be made as small as we please by taking $x$ sufficiently large, (I mean by taking $x$ greater than some certain value $\xi$ ). Then

$$
\begin{aligned}
\left(1+\epsilon_{x}\right) \log 2 . x<\psi x-\psi \frac{x}{2}+\psi \frac{x}{3} \ldots-\psi \frac{x}{2 m} & \\
<Q(1+h) x\left(1-\frac{1}{2}\right. & \left.+\frac{1}{3} \ldots-\frac{1}{2 m}\right) \\
& +F x-F \frac{x}{2}+F \frac{x}{3} \ldots-F \frac{x}{2 m}
\end{aligned}
$$

Let $\xi$ be taken so great that for all values of $x$ greater than $\frac{\xi}{2 m}, \frac{F x}{x}$ shall be less in absolute numerical value than $\frac{k}{2 m}$, where $k$ is an arbitrary positive quantity: then, if we take $x>\xi$, the sum of the absolute values of $F x, F \frac{x}{2}, F \frac{x}{3}, \ldots F \frac{x}{2 m}$, is less than $k x$; and $\grave{a}$ fortiori

$$
F x-F \frac{x}{2}+F \frac{x}{3} \ldots-F \frac{x}{2 m}<k x .
$$


Therefore

$$
Q(1+h) \log 2 . x>\left(1+\epsilon_{x}\right) \log 2 . x-k x .
$$

Hence, $Q$ being greater than $\frac{1+\epsilon_{x}}{1+h}-\frac{k}{(1+h) \log 2}$, and $\epsilon_{x}, h, k$ being all three capable of becoming indefinitely small, $1-Q$ cannot be a finite positive quantity; which amounts to saying that $1-Q$ cannot be positive.

In precisely the same manner, dealing with the other limit to $V x$ and stopping in its development at the term $\psi \frac{x}{2 m+1}$ (instead of stopping at the term $\left.-\psi \frac{x}{2 m}\right)$ it may be proved that $1-Q$ cannot be negative. Hence $1-Q$ must be zero, that is, $Q=1$. Q. E. D.

We have thus determined what is the common limit to which the principal terms in the superior and in the inferior limits of $\psi x$ are bound to approximate, on the supposition of the possibility of formulae being discoverable admitting of the interval between these principal terms being capable of being made as small as we please. But to pronounce with certainty upon the existence of such possibility, we shall probably have to wait until some one is born into the world as far surpassing Tchebycheff in insight and penetration as Tchebycheff has proved himself superior in these qualities to the ordinary run of mankind. 